\title{
Establishment of a transgenic mouse model of corneal dystrophy overexpressing human BIGH3
}

\author{
XIN LIAO $^{1}$, HONGPING CUI $^{2}$ and FANG WANG ${ }^{1}$ \\ ${ }^{1}$ Department of Ophthalmology, Shanghai Tenth People's Hospital Affiliated to Tongji University, \\ Shanghai 200072; ${ }^{2}$ Department of Ophthalmology, Shanghai East Hospital Affiliated to \\ Tongji University School of Medicine, Shanghai 200120, P.R. China
}

Received May 16, 2013; Accepted August 22, 2013

DOI: $10.3892 /$ ijmm.2013.1480

\begin{abstract}
This study aimed to establish a transgenic mouse model of corneal dystrophy (CD) overexpressing the human transforming growth factor, $\beta$-induced, $68 \mathrm{kDa}$ (TGFBI, also known as $B I G H 3$ ) gene. A purified and linearized recombinant plasmid carrying the expression cassette $B I G H 3$-IRES-EGFP was microinjected into the pronuclei of $\mathrm{C} 57 \mathrm{BL} / 6 \mathrm{~J}$ mouse fertilized eggs under the control of the phosphoglycerate kinase (PGK) promoter. The expression of human BIGH3 in the transgenic mice was confirmed by PCR using DNA extracted from tail tissue. Four founder transgenic mice were identified by PCR and the increased expression of BIGH3 was observed in the corneas of the transgenic mice by RT-PCR and western blot analysis. The abnormal corneas with central opacity were observed in the transgenic mice by corneal photography. We concluded that the exogenous gene, $B I G H 3$, was integrated successfully into the mouse genome through microinjection. In addition, the phenotype observed in this BIGH3 transgenic mouse model was similar to $\mathrm{CD}$. Therefore, this transgenic model may prove useful in the investigation of the pathogenesis of CD.
\end{abstract}

\section{Introduction}

Human corneal dystrophy (HCD) is a group of genetic disorders characterized by a non-inflammatory, bilateral opacity of the cornea (1). Many types of HCD are associated with transforming growth factor, $\beta$-induced, $68 \mathrm{kDa}$ (TGFBI, also known as BIGH3) gene mutations. The symptoms may appear before the age of 20 , developing during adolescence and gradually progressing throughout life. HCD often affects only one layer of the cornea at first and then the disease

Correspondence to: Professor Fang Wang, Department of Ophthalmology, Shanghai Tenth People's Hospital Affiliated to Tongji University, 301 Middle Yan Chang Road, Shanghai 200072, P.R. China

E-mail: 18917683335@163.com

Key words: BIGH3, corneal dystrophy, transgenic mice progresses to the remainder of the cornea. Histopathological studies have also proven that the BIGH3 protein can be found in these corneal deposits. Penetrating keratoplasty has commonly been performed for extensive CD. However, recurrence and deterioration of the disease has often been observed even after surgery (2).

Although studies of $B I G H 3$-related $\mathrm{HCD}$ are of great interest $(3,4)$, the role of $B I G H 3$ mutations in HCD remains to be fully understood due to the lack of animal models (5). Since there are many limiting factors in the study of HCD, relevant animal models would greatly contribute to the study of this pathogenesis. Transgenic mice produced by microinjection are the most widely used animal models. Therefore, in this study, a transgenic mouse model expressing the human $B I G H 3$ gene was established. Our data also indicated that the human $B I G H 3$ gene was overexpressed in the mouse corneas and induced corneal opacity in the eyes of the mice.

\section{Materials and methods}

Animals. C57BL/6J mice were purchased from the Shanghai Biomodel Research Center (Shangai, China). All animals were fed with sterile mouse food and water at $\mathrm{pH}$ 2.8-3.2 under specific pathogen-free (SPF) conditions and were kept in an isolated room with an automatic light control (14 h day light and $10 \mathrm{~h}$ dark cycle). The animal experiments were approved by the Institutional Animal Care and Use Commitee (IACUC) of the Shangai Biomodel Research Center for Experimental Animal Management (permit no. IACUC 2010-0001). All animal experiments were conducted in compliance with the relevant provisions of the Association for Research in Vision and Ophthalmology (ARVO; American Association of Ophthalmology) for animal research.

Construction of the pPGK-BIGH3-IRES-EGFP transgene vector. A $0.5 \mathrm{~kb}$ phosphoglycerate kinase (PGK) promoter fragment was amplified by PCR using DNA polymerase Ex Taq (Takara Bio, Inc., Shiga, Japan) and pPL451 as a template (Shanghai Biomodel Research Center). The forward and reverse primers were as follows: 5'-CGACTCGAGACC GGGTAGGGGAGGCGCTTT-3' and 5'-GGCGTCGACTCG AAAGGCCCGGAGATGAGG-3', respectively. The amplicon flanked with $\mathrm{XhoI}$ and $\mathrm{SalI}$ was recovered from the agarose gel 
and ligated into the similarly double digested plasmid, pCAG-IRES-EGFP, which was obtained from the same supplier. The ligation was transformed into E. coli in order to isolate the recombinant plasmid, pPGK-CAG-IRES-EGFP. The plasmid DNA was digested with $P h B I$ and purified to remove the CAG promoter fragment by agarose gel electrophoresis. The recovered DNA was self-ligated to generate the recombinant plasmid, pPGK-IRES-EGFP.

The cDNA fragment of BIGH3 (Source BioScience, Nottingham, UK) was digested with SalI and SacII and ligated to the similarly digested pPGK-IRES-EGFP. The ligation mixture was transformed into $E$. coli to obtain the recombinant plasmid, pPGK-BIGH3-IRES-EGFP. The plasmid was sequenced for confirmation before being linearized with $X b a I$, purified with phenol-chloroform extraction and used in microinjection buffer $(\mathrm{pH} 7.4)$ at a concentration of $4 \mathrm{ng} / \mu \mathrm{l}$ to produce the transgenic mice.

Generation of the transgenic animals. The purified pPGK-BIGH3-IRES-EGFP plasmid DNA was microinjected into the fertilized eggs of $\mathrm{C} 57 \mathrm{BL} / 6 \mathrm{~J}$ mice. After the injection, the fertilized eggs that were in a good condition were transplanted into the fallopian tubes of pseudo-pregnant female mice of the same strain, to produce the $\mathrm{F}_{0}$ generation animals. All animals were kept in an isolated room at $22^{\circ} \mathrm{C}$, with an automatic light control ( $14 \mathrm{~h}$ day and $10 \mathrm{~h}$ dark cycle). The $\mathrm{F}_{0}$ animals were mated with the wild-type mice to generate $F_{1}$ animals.

Genomic DNA extraction and PCR genotyping. The transgenic founders and offsprings were identified by PCR of the genomic DNA extracted from tail biopsies. The tissues were digested overnight in a water bath at $55^{\circ} \mathrm{C}$ in $500 \mu \mathrm{l}$ of a lysis buffer, containing $20-100 \mathrm{mg} / \mathrm{ml}$ proteinase $\mathrm{K}, 50 \mathrm{mmol} / 1$ Tris, $\mathrm{pH} 8.0$, $100 \mathrm{mmol} / \mathrm{l}$ EDTA, $100 \mathrm{mmol} / \mathrm{l} \mathrm{NaCl}$ and 1\% SDS (Bio-Rad, USA). The digested tissues were extracted twice with a double volume of phenol-chloroform and the supernatants were diluted in a double volume of absolute ethanol to precipitate the DNA. After having been washed twice with $70 \%$ ethanol, the pellets were air-dried and dissolved in $100 \mu 1 \mathrm{TE}$ buffer. The DNA concentration was determined using a UV spectrophotometer. PCR was conducted to confirm the presence of the exogenous gene in pPGK-BIGH3-IRES-EGFP using the forward primer, 5'-GACTAGCCCCTGTCTATCAAAAGTT-3' and the reverse primer, 5'-AACCTCGACTAAACACATGTAAAGC-3', which produced a product of $578 \mathrm{bp}$. PCR was carried out under the following conditions: $95^{\circ} \mathrm{C}$ for $3 \mathrm{~min}$, followed by 25 cycles of $95^{\circ} \mathrm{C}$ for $15 \mathrm{sec}, 65^{\circ} \mathrm{C}$ for $30 \mathrm{sec}, 72^{\circ} \mathrm{C}$ for $1 \mathrm{~min}$ and with an extension at $72^{\circ} \mathrm{C}$ for $10 \mathrm{~min}$ at the end of the reaction. The results of the reaction were visualized by gel electrophoresis. Wild-type DNA was run as a negative control on a $1 \%$ agarose gel electrophoresis.

RT-PCR of human BIGH3 expression in the cornea. Several corneas were removed from two randomly selected wild-type and PCR-positive $F_{1}$ transgenic mice, which were euthanized, irrespective of their gender. Euthanasia was performed by cervical dislocation. Total RNA was extracted using TRIzol reagent and digested with DNase. Phenol-chloroform was used to extract the residual genomic DNA before being subjected to reverse transcription. cDNA was obtained using an RT-PCR kit and Gapdh as the control. The following PCR primers were used for RT-PCR: Gapdh forward, 5'-TGGGAAGCTGGTCATCAAC-3' and reverse, 5'-GCATCACCCCATTTGATGTT-3'; and BIGH3 forward, 5'-CAGGCGTCAGCGTATTCC-3' and reverse, 5'-CCTTCCCTACCCGTCCAA-3'. PCR was conducted under the following conditions: $90^{\circ} \mathrm{C}$ for $30 \mathrm{sec}, 61^{\circ} \mathrm{C}$ for $20 \mathrm{~min}$ followed by pre-denaturation at $94^{\circ} \mathrm{C}$ for $30 \mathrm{sec}$ and 35 cycles of amplification at $55-61^{\circ} \mathrm{C}$ for $30 \mathrm{sec}, 72^{\circ} \mathrm{C}$ for $30 \mathrm{sec}$ and then extension at $72^{\circ} \mathrm{C}$ for $5 \mathrm{~min}$. The products were analyzed on a $1 \%$ agarose gel. All experiments were repeated three times independently.

Western blot analysis of human BIGH3 expression in the cornea. Three wild-type mice served as the controls. Corneas were collected under a stereo microscope from the eye balls of euthanized mice following cervical dislocation, each randomly selected from wild-type and PCR-positive $F_{1}$ transgenic mice. The corneal tissues were separated using micro-tweezers, weighed and cut into small sections using micro scissors. The tissues were added with equal volumes of lysis buffer, homogenized on ice and incubated for $30 \mathrm{~min}$ at $4^{\circ} \mathrm{C}$ in a refrigerator. The digested tissues were transferred to $1.5 \mathrm{ml}$ centrifugation tubes and spun for $1 \mathrm{~min}$ at $12,000 \mathrm{rpm}$ at $4^{\circ} \mathrm{C}$. The supernatants were heated for $5 \mathrm{~min}$ in a water bath at $100^{\circ} \mathrm{C}$ before being used for SDS-PAGE. The proteins were blotted onto nitrocellulose membranes following electrophoresis and reacted with 1:500 diluted antibodies against BIGH3 (Abcam, Cambridge, UK) to detect the expression product. The images were scanned using the LI-COR Odyssey ${ }^{\circledR}$ Infrared Laser Imaging System (LI-COR Biotechnology; Lincoln, NE, USA). The results were expressed as a percentage of the control optical density. All experiments were repeated three times.

Corneal photography. The corneas of these transgenic mice were photographed using a digital camera following anesthesia to determine the gross appearance of the corneas.

Statistical analysis. Data were statistically analyzed using SPSS 17.0 software (SPSS, Chicago, IL, USA). Quantitative data were tested using the F-test for homogeneity of variance. Data with homogeneous variance were tested using the independent sample t-test, while non-homogeneous data were analyzed by the Mann-Whitney U test. A p-value $<0.05$ was considered to indicate a statistically significant difference.

\section{Results}

Construction of PGK-BIGH3-IRES-EGFP vector. The PGK promoter fragment and the XhoI and SalI digested plasmid, pCAG-IRES-EGFP, were ligated to produce pPGK-CAG-IRES-EGFP. The plasmid was digested and gel purified to remove the CAG fragment and then self-ligated to obtain pPGK-IRES-EGFP. Human BIGH3 cDNA was cloned into the $S a l \mathrm{I} / \mathrm{Sac}$ II site of pPGK-IRES-EGFP to generate a new 7416 bp recombinant vector, pPGK-BIGH3-IRES-EGFP.

Identification offounder transgenic mice. Following pre-nuclei microinjection of the linearized plasmid pPGK-BIGH3IRES-EGFP, 36 founder mice were generated. PCR indicated 


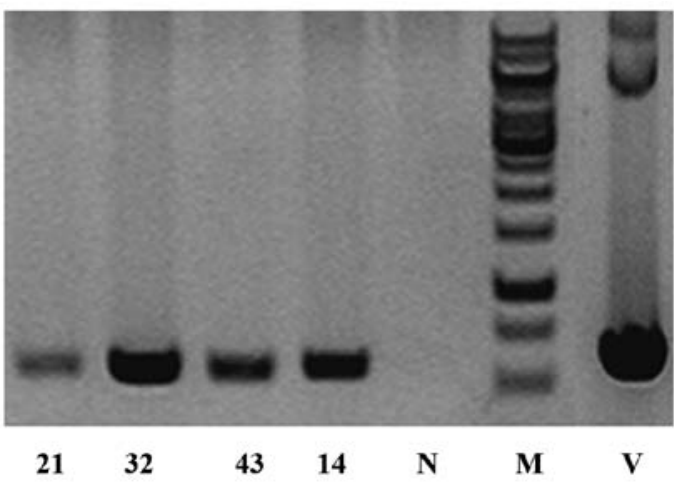

Figure 1. Agarose gel electrophoresis of PCR products on genomic DNA from mouse tails. The PCR products from transgenic mice, numbered 21, 32,43 and 14, are shown in lanes 1-4, respectively; the PCR products using wild-type mouse DNA as templates are shown as negative $(\mathrm{N})$; the $1 \mathrm{~kb}$ DNA ladder as $(\mathrm{M})$ and the PCR product with the transgenic vector $(\mathrm{V})$ as a template is shown as positive.

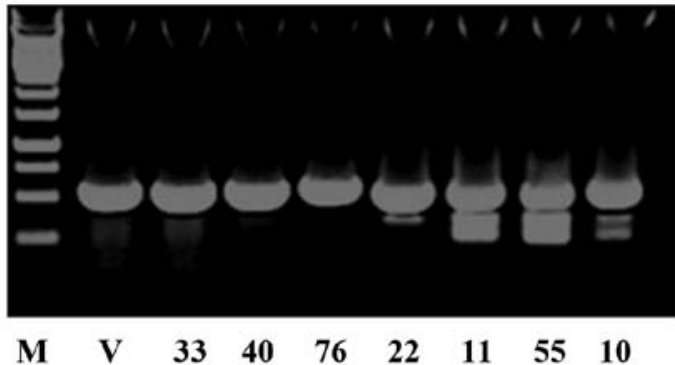

Figure 2. Identification of $\mathrm{F}_{1}$ transgenic mice by PCR. The $1 \mathrm{~kb}$ DNA ladder is shown as a marker $(\mathrm{M})$; the PCR product with the transgenic vector $(\mathrm{V})$ as positive; the PCR products from transgenic mice, numbered 33, 40, 76, 22, 11,55 and 10, are shown in lanes 3-9, respectively.

that four of them (M21, male; M32, female; M43, female; M14, male) were transgenic founders. The PCR-positive animals were confirmed twice along with three wild-type animals using DNA prepared from the sampled tail tips with an amplified product of $578 \mathrm{bp}$ in the transgenic but not in the wild-type mouse samples (Fig. 1).

Generation of $F_{1}$ transgenic mice. The four $\mathrm{F}_{0}$ transgenic mice were mated with wild-type $\mathrm{C} 57 \mathrm{BL} / 6 \mathrm{~J}$ mice to produce $\mathrm{F}_{1}$ animals and the offsprings were identified by PCR genotyping ten days after birth. A total of 30 mice were born. PCR analysis indicated that seven of them [M33, female; M40, female; M76, male; M22, male; M11, female; M55, female; M10, male] were transgenic (Fig. 2).

Gross phenotype of transgenic mice. The corneas of these transgenic mice were photographed using a digital camera following anesthesia to determine the gross appearance of the corneas. There were five transgenic mice among the seven $F_{1}$ transgenic mice, that displayed a centrally reduced corneal transparency, which was visible when the eyelids opened at approximately two weeks after birth (Fig. 3).

$R T$-PCR of corneal tissues from $F_{1}$ transgenic mice. The corneas were removed from the dissected $\mathrm{F}_{1}$ transgenic mouse
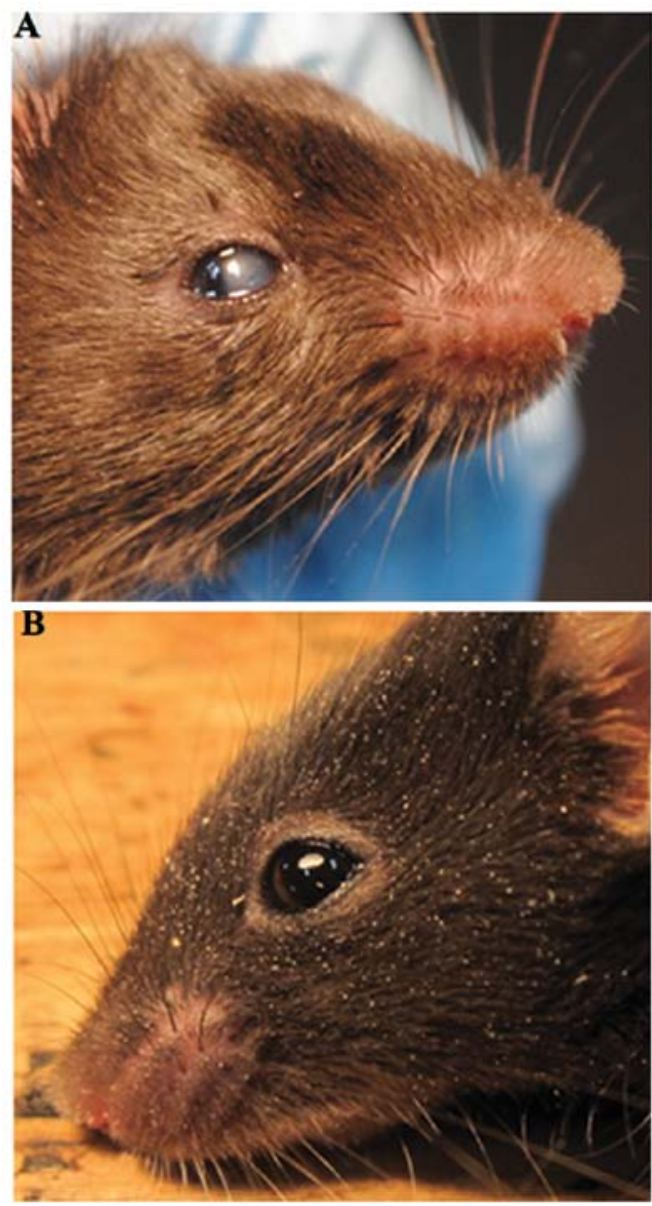

Figure 3. Gross phenotype of the wild-type and $B I G H 3$ transgenic mice. The (A) transgenic mice showed corneal opacity compared with the (B) transparent cornea of the wild-type animals.

eyes. RT-PCR was performed to examine the corneas of two $\mathrm{F}_{1}$ transgenic mice in order to investigate the relative expression of human BIGH3 mRNA in the corneas from wild-type and transgenic mice. As illustrated in Fig. 4, compared with the wild-type mice, the expression of BIGH3 in the transgenic mice was significantly upregulated in their corneas $(\mathrm{p}<0.01)$.

Western blot analysis of the corneas of BIGH3 transgenic mice. Western blot analysis was performed to examine the corneas of three $F_{1}$ transgenic mice in order to investigate the expression of the human BIGH3 gene in the corneas from wild-type and transgenic mice. As the anti-human BIGH3 antibody partially cross-reacted with both the mouse and human proteins, the amount of BIGH3 in the wild-type mice likely represented the level of endogenous mouse BIGH3 in the corneas. As illustrated in Fig. 5, in comparison with the wild-type mice, the transgenic mice had higher levels of BIGH3 in their corneas $(\mathrm{p}<0.01)$.

\section{Discussion}

BIGH3 (68 kDa), also known as TGFBI, is located on chromosome $5 \mathrm{q} 31$, spanning a region of $30 \mathrm{~kb}(6)$. A mutation was initially discovered when TGF was used to treat adenocarcinoma cell lines (7-9). Research to date on BIGH3 has focused on two point mutations of arginine residues 124 and $555(10,11)$. 

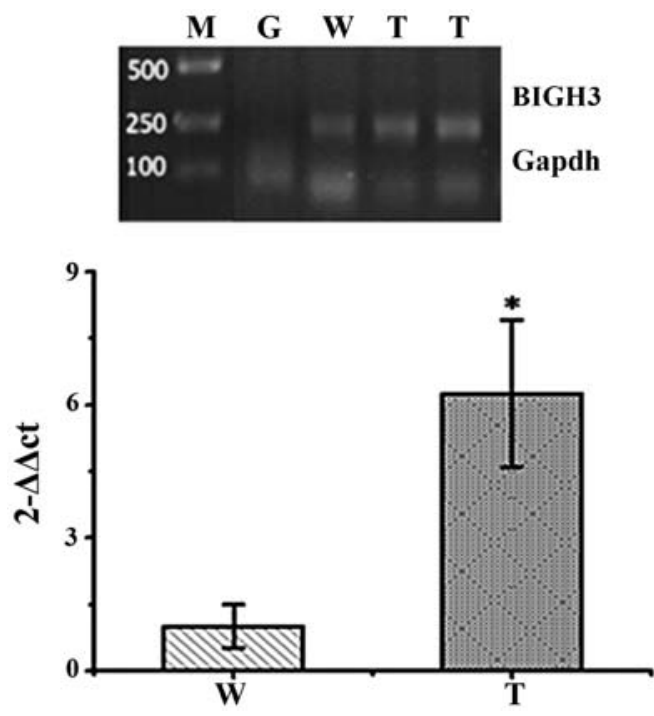

Figure 4. RT-PCR of the human $B I G H 3$ gene in corneas from wild-type (W) and transgenic (T) mice. A higher expression of the human $B I G H 3$ gene (242 bp) was detected by RT-PCR in the corneas of transgenic mice compared with the wild-type mice. Gapdh (78 bp) was used as an internal control. M, DNA size marker; G, Gapdh; " $p<0.01$.

They are the most common mutations in the human population (12). Few reports are available regarding the establishment of animal models for CD. Kim et al (13) reported the use of the $\mathrm{Alb}$ promoter to establish a transgenic mouse model of $\mathrm{CD}$ overexpressing $B I G H 3$. However, the mice were subsequently found to have anterior segment disease, including corneal opacity, cataract and iris abnormalities. Bustamante et al (14) also described the development of the model with the mutated BIGH3 gene R555W using lentiviral vectors. However, they found that the animals tended to age more rapidly, with retinal degeneration, although their corneas were normal.

To achieve a high level of expression of exogenous genes and efficient secretion in mammalian cells, it is necessary to use suitable vectors and recipient systems that enable the effective induction of expression. The key element is a strong promoter to drive the expression cassette. In this study, we used the PGK promoter to drive the high level expression of the BIGH3 gene. The PGK promoter has been widely used in yeast expression systems $(15,16)$ and is recognized as a strong constitutive promoter with a wide range of hosts. It has three transcriptional start sites with a high GC content. The GC content upstream of the transcriptional start sites can reach $70 \%$. It does not contain conserved sequences, such as TATA or CAAT boxes as often observed in other promoters. There is a repeat sequence GGGGCGG upstream from the transcriptional start sites $(17,18)$. All these features describe the high efficiency of the PGK promoter and the reasons we selected it.

The pCAG-IRES-EGFP vector, also used in this study, has an enhanced GFP gene with an internal ribosomal entry site (IRES). GFP is non-toxic, stable and easy to visualize (19). It has been widely used to monitor transfection efficiency, expression levels and to sort the transfected cells. IRES is a conserved cis-acting element existing in the mRNA of eukaryotes or viral genomes. It binds to ribosomes to mediate the 5 ' cap-dependent translation of downstream genes. The sequence has been
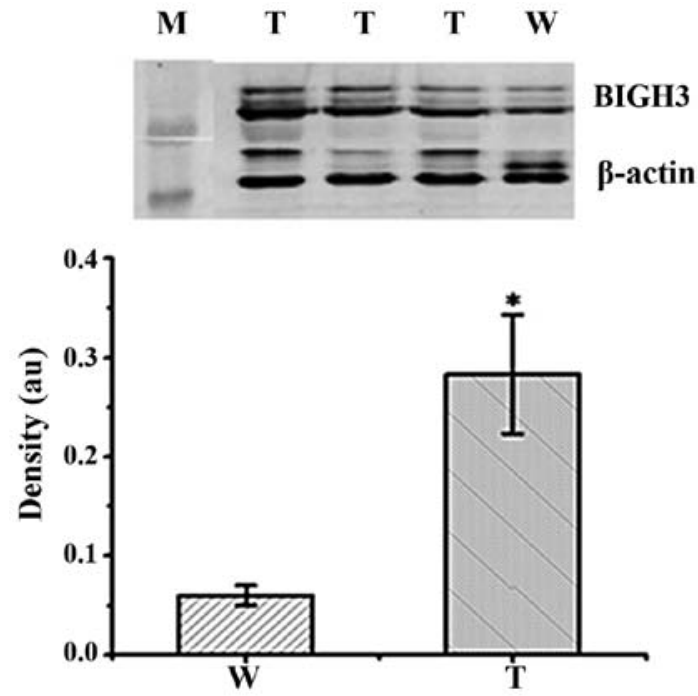

Figure 5. Western blot analysis of the human BIGH3 protein in the corneas from wild-type $(\mathrm{W})$ and transgenic $(\mathrm{T})$ mice. $\beta$-actin protein $(42 \mathrm{kDa})$ was used as an internal protein control. The $B I G H 3$ protein $(68 \mathrm{kDa})$ was significantly increased in the corneas of the transgenic mice compared with those of the wild-type mice. The results are expressed as the means \pm SD. M, marker; * $\mathrm{p}<0.01$.

widely used in various binary expression vectors (20-22). In our study, we intended to use EGFP as a reporter to measure the expression of the $B I G H 3$ transgene. However, the GFP fluorescence observed in vivo under a fluorescence microscope was too weak to fulfill this purpose. We speculate that this may be due to the dark fur color of the C57BL/6J mice, leading to the absorption of most of the GFP fluorescence.

Male pronuclear microinjection was applied to generate transgenic mice in our study, which is currently the most commonly used method for transgenic mouse production. The recombinant plasmid, PGK-BIGH3-IRES-EGFP, was injected into the pronuclei of 85 fertilized eggs of C57BL/6J mice to produce $B I G H 3$ transgenic mice. Four transgenic founder mice (M21, male; M32, female; M43, female; M14, male) of 36 born mice were successfully generated, as confirmed by PCR analysis. After mating with wild-type C57BL/6J mice, a total of $30 \mathrm{~F}_{1}$ offspring mice were produced and seven of them were PCR-positive (M33, female; M40, female; M76, male; M22; male, M11; female, M55; female, M10; male), indicating that the human $B I G H 3$ gene had integrated into the mouse genome and was able to be transmitted to the next generation. However, we also noted that only $6-25 \%$ of the transgenic mice were able to transmit the $B I G H 3$ gene to the next generation, which is a low integration rate. We speculate that this is due to the multiple integration and chimerical structure of the foreign genes.

Subsequently, we observed that the $B I G H 3$ transgenic mice did not present apparent macroscopic abnormalities compared with the wild-type mice, suggesting that $B I G H 3$ overexpression did not severely impair mouse development. A digital camera was also applied to determine the gross appearance of the corneas of these transgenic mice following anesthesia. There were five among the seven $F_{1}$ transgenic mice that displayed centrally reduced corneal transparency, as illustrated in Fig. 3, which was similar to the symptoms of HCD. 
Furthermore, RT-PCR and western blot analysis were also performed with the corneas of $F_{1}$ transgenic mice to investigate the expression of the human $B I G H 3$ gene in the corneas from wild-type and transgenic mice. As the anti-human BIGH3 antibody partially cross-reacted with both mouse and human proteins, the amount of BIGH3 in the wild-type mice likely represented the level of the endogenous mouse BIGH3 in the corneas. We demonstrated that, in comparison with the wild-type mice, the transgenic mice had a significantly increased expression of BIGH3 in their corneas (Fig. 5).

Taken together, the results from our study suggest that we successfully generated a transgenic mouse model overexpressing the human $B I G H 3$ gene under the control of the PGK promoter, which may serve as a practical model for studying HCD. In addition, we believe that the $B I G H 3$ gene is essential for ocular development in vivo and pathologically important to corneal disorganization. Therefore, this transgenic mouse model is likely to contribute to further studies concerning the detailed pathogenesis of the overexpression of $\mathrm{BIGH} 3$ protein in corneal dystrophy.

\section{Acknowledgements}

The present study was supported by a grant from the experimental animal project of the Shanghai Science and Technology Commission (no. 10140903900).

\section{References}

1. Eiberg H, Moller HU, Berendt I and Mohr J: Assignment of granular corneal dystrophy Groenouw type I (CDGG1) to chromosome 5q. Eur J Hum Genet 2: 132-138, 1994.

2. Yu P, Gu Y, Yang Y, et al: A clinical and molecular-genetic analysis of Chinese patients with lattice corneal dystrophy and novel Thr538Pro mutation in the TGFBI (BIGH3) gene. J Genet 85: 73-76, 2006.

3. Paliwal P, Sharma A, Tandon R, et al: TGFBI mutation screening and genotype-phenotype correlation in north Indian patients with corneal dystrophies. Mol Vis 16: 1429-1438, 2010.

4. Vincent AL, de Karolyi B, Patel DV, et al: TGFBI mutational analysis in a New Zealand population of inherited corneal dystrophy patients. Br J Ophthalmol 94: 836-842, 2010.

5. Korvatska E, Munier FL, Djemai A, et al: Mutation hot spots in 5q31-linked corneal dystrophies. Am J Hum Genet 62: 320-324, 1998.

6. Skonier J, Bennett K, Rothwell V, et al: Beta-igh3: a transforming growth factor-beta responsive gene encoding a secreted protein that inhibits cell attachment in vitro and suppresses the growth of CHO cells in nude mice. DNA Cell Biol 13: 571-584, 1994.
7. Grunauer-Kloevekorn C, Braeutigam S, Weidle E, et al: Molecular genetic and histopathological examinations for genotype-phenotype analysis in patients with TGFBI-linked corneal dystrophy. Klin Monbl Augenheilkd 223: 829-836, 2006 (In German).

8. Kannabiran C and Klintworth GK: TGFBI gene mutations in corneal dystrophies. Hum Mutat 27: 615-625, 2006.

9. Patel DA, Chang SH, Harocopos GJ, et al: Granular and lattice deposits in corneal dystrophy caused by R124C mutation of TGFBIp. Cornea 11: 1215-1222, 2010.

10. Gruenauer-Kloevekorn C, Clausen I, Weidle E, et al: TGFBI (BIGH3) gene mutations in German families: two novel mutations associated with unique clinical and histopathological findings. Br J Ophthalmol 93: 932-937, 2009.

11. Grunauer-Kloevekorn C, Brautigam S, Wolter-Roessler M, et al: Molecular genetic analysis of the BIGH3 gene in lattice type I (Biber-Haab-Dimmer) and granular type II (Avellino) corneal dystrophy: is indirect mutation analysis for hot spots recommended? Klin Monbl Augenheilkd 222: 1017-1023, 2005 (In German).

12. Munier FL, Korvatska E, Djemai A, et al: Kerato-epithelin mutations in four 5q31- linked corneal dystrophies. Nat Genet 15: 247-251, 1997.

13. Kim JE, Han MS, Bae YC, et al: Anterior segment dysgenesis after overexpression of transforming growth factor-beta-induced gene, beta igh3, in the mouse eye. Mol Vis 13: 1942-1952, 2007.

14. Bustamante M, Tasinato A, Maurer F, et al: Overexpression of a mutant form of TGFBI/BIGH3 induces retinal degeneration in transgenic mice. Mol Vis 14: 1129-1137, 2008.

15. Pey AL, Mesa-Torres N, Chiarelli LR and Valentini G: Structural and energetic basis of protein kinetic destabilization in human phosphoglycerate kinase 1 deficiency. Biochemistry 52: 1160-1170, 2013.

16. Schay G, Herenyi L, Fidy J and Osvath S: Role of domain interactions in the collective motion of phosphoglycerate kinase. Biophys J 104: 677-682, 2013.

17. Lei Y and Liu YG: Isolation, sequence identification and tissue expression profile of a novel sheep gene-PGK1. Res J Biotechnol 8: 38-41, 2013.

18. Singer-Sam J, Keith DH, Tani K, et al: Sequence of the promoter region of the gene for human X-linked 3-phosphoglycerate kinase. Gene 32: 409-417, 1984.

19. Takada T, Lida K, Awaji T, et al: Selective production of transgenic mice using green fluorescent protein as a marker. Nat Biotechnol 15: 458-461, 1997.

20. Mizuguchi H, Xu Z, Ishii-Watabe A, et al: IRES-dependent second gene expression is significantly lower than cap-dependent first gene expression in a bicistronic vector. Mol Ther 1: 376-382, 2000.

21. Borman AM, Bailly JL, Girard M and Kean KM: Picornavirus internal ribosome entry segments: comparison of translation efficiency and the requirements for optimal internal initiation of translation in vitro. Nucleic Acids Res 23: 3656-3663, 1995.

22. Cao HQ and Ding JF: Construction strategy of polygene co-expression vectors. Foreign Med Sci (Molecular Biology Section) 24: 1-4, 2000. 